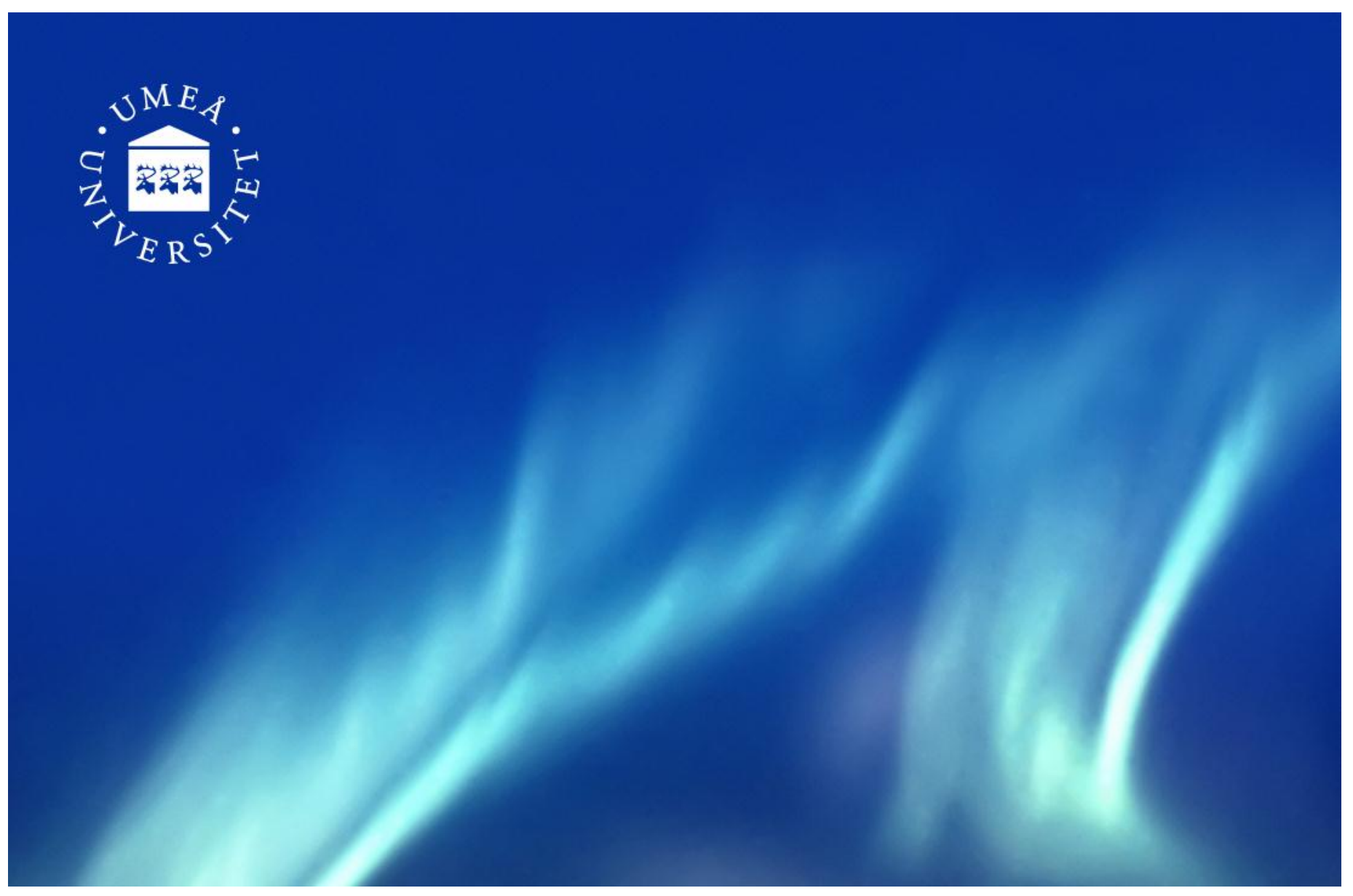

DiVA - Digitala Vetenskapliga Arkivet http://umu.diva-portal.org

This is an author produced version of a paper published in:

Qualitative Research in Psychology

This paper has been peer-reviewed but does not include the final publisher proof-corrections or journal pagination.

Citation for the published paper:

Ingrid Schéle, Leif Hedman, Anne Hammarström

Shared ambiguity but different experiences and demands among dental students - a gender perspective

In: Qualitative Research in Psychology, Vol. 8 (2011):1, s. 1-25.

DOI: $10.1080 / 14780880902874231$

Access to the published version may require subscription. Published with permission from:

Routledge, Taylor \& Francis Group 


\title{
Shared ambiguity but different experiences and demands among dental students - a gender perspective
}

\author{
Authors: \\ Ingrid Schéle, Department of Psychology, Umeå University, Sweden \\ (corresponding author) \\ E-mail: ingrid.schele@psy.umu.se \\ Leif Hedman, Department of Psychology, Umeå University \\ Anne Hammarström, Department of Family Medicine, Umeå University
}

\section{About the authors}

Ingrid Schéle holds a degree in Human Resource management and is currently a Doctoral student at the Department of Psychology and the Umeå Centre for Gender Studies, the Graduate School for Gender Studies, both at Umeå University, Sweden. Her research interests are the dual role of dental students, who treat patients both in order learn and in order to treat, as well as the feminisation of the formerly male domain of dentistry.

Leif Hedman is a licensed psychologist and Professor in psychology at the Department of Psychology, Umeå University. Since 2003 he is also an affiliated researcher within the fields of Medical simulation and Medical Human Factors at the Center for Advanced Medical Simulation at Karolinska Institutet, Stockholm, Sweden, where he conducts research in the areas of context specific medical (simulator-based) training and the transfer of learning. Since 2002 he has as a principal investigator and senior researcher an international and interdisciplinary collaboration with researchers in medicine at Stanford University, USA.

Anne Hammarström is since 2000 Professor of Public Health at Umeå University and also a General Practitioner. One of her main research areas is gender research in public health and since 2004 she leads Umeå Centre for Gender Studies in Medicine. Since 1981 she is the principal investigator of the Northern Swedish Cohort study of school leavers. She has a broad international and interdisciplinary collaboration, with among others researchers in psychology, medical sociology and public health in Europe as well as in Australia. 


\begin{abstract}
This study explores how dental students experience their clinical semesters from a gender perspective.

Twelve students (seven women and five men) and three teachers (two women and one man) at the Umeå dentistry programme participated in semistructured interviews that were analysed with Grounded Theory methodology.

The model we propose consists of the core category Experiencing ambiguity and the three categories Experiencing pressure and stress, Assessing your own performance, and Passing through the eye of the needle and also includes four subcategories. At the core of our findings lies ambiguity, captured in the student dilemmas What's enough/When's enough. The answers to these dilemmas are further complicated by the gendered dimension and the dimension of unequal treatment, which provide students with different and contradicting sets of rules and roles. A comparison with recent findings from the U.S. shows that their experiences are not unique.

Our Experiencing ambiguity model constitutes a platform for future research on how students experience clinical education, as well as potential predictors and consequences in relation to performance and well-being.
\end{abstract}

Keywords: dental students, gender perspective, psychosocial work environment, grounded theory, ambiguity, control 


\section{Introduction}

The lack of studies that explore or investigate dental education from a student perspective has recently attracted multinational attention within the field of dental education (Divaris et al., 2008; Henzi et al., 2005; Henzi, Davis, Jasinevicius \& Hendricson, 2006; Henzi, Davis, Jasinevicius \& Hendricson, 2007). In a call for action Divaris and his collaborators stress both the uniqueness of dental education and the similarities in dental students' experiences around the world. Divaris et al. proclaim that clinical training is the most important part of the programme, as this is when students are introduced to the practical side of their future profession. Studies suggest that clinical training has both positive and negative connotations - it can relieve students of (anticipatory) stress (Humphris et al., 2002; Polychronopoulou \& Divaris, 2005), but it can also cause stress (Henzi et al., 2007). Henzi et al. also noted that junior students were most concerned with faculty supportiveness, possibly in relation to teaching and learning issues in the clinic (2005).

Furthermore, few studies have applied a gender perspective (e.g. Freeman, Gorter, McWilliams \& Williams, 2007). Applying a gender perspective entails the analysis of individual, social and structural relations between the sexes from a social (constructionist) and historical standpoint. In accordance with this standpoint our study focuses on how students experience the Umeå dentistry programme rather than measurements of their inner states, traits or, for example, hours spent studying. Our main reason for exploring dental education from an experiential gender perspective is the ongoing shift in the student population.

Historically dentistry has been a male profession in Western Europe and North America, but women have entered the profession in increasing numbers from the 1960s and onwards. Even though statistics are somewhat incomplete, approximately 36 percent of the dentists in these regions were women in the year 2000 (Zillén \& Mindak, 2000). The number of female dentists is expected to rise due to a recent, marked, shift in the gender distribution of the dental student population in Western Europe and North America; where the majority of the students now are women (Nunn et al., 2008). After a dip in the 1980-90s characterised by few applicants per place, the dentistry programme is re-establishing its status as one of the most popular higher educational programmes in Sweden, which has led to strict admittance requirements. In the period 2002-2006 69 percent of the female students were admitted to the dentistry programmes based on credits 
received at upper secondary school, compared to 47 percent of their male peers (Statistics Sweden, 2007).

In the light of the increasing number of women who enter a setting where men have previously constituted the majority, the question arises whether the women are expected to adhere to the norm set by male students/dentists, or if they are expected to become a different kind of student/dentist. Research in the U.S. suggests that the male norms for dental students/dentists that dominated in the 1970s has shifted to include both men and women (Rosenberg, Cucchiara \& Helpin, 1998), but female students may still be at a disadvantage vis-à-vis dental nurses who, with few if any exceptions, are women (Freeman et al., 2007). Studies on the relation between medical doctors and nurses suggest that female doctors are expected to adhere to male professional norms in order to gain respect and female gender norms to gain social acceptance, which can lead to a situation where the interaction and communication between doctors and nurses is characterised by conflicting demands (e.g. Cassell, 1997; Davies, 2001; Eriksson, 2003).

In this study we use Connell's (2002) relational theory of gender as our analytical framework. This theory contains four dimensions: power relations, production relations, emotional relations and symbolic relations. As the medical profession has been dominated by men, and medical hierarchical institutions have been based on men as the norm or ideal (Davies, 2001; Eriksson, 2003), it is of interest to search for remaining traces of institutionalised power relations in our material. These traces may well appear in the form of a more diffuse, discursive power (Connell, 2002). In analogy with this previous research it is also possible that female students are expected or expect to become a different kind of dentist than their male peers, which might structurally tilt the dental profession towards the female work domain. As research has found indications of flirting between nurses and students/doctors in medical as well as dental settings (Davies, 2001; Freeman et al., 2007), we also believe that these kinds of interaction will benefit from an analysis along the dimension of emotional relations. Furthermore, Symbolic relations may in this context relate to the male history of dentistry, as a female dental student adorning the white coat transgresses a (blurred) boundary and may need to relate to the (male) attributes that come with it.

In this study we will explore, from a gender perspective, how dental students experience their education. We will focus on the clinical semesters 
(semesters 5-10) as they seem to be the most critical and important period of the dental programme. Our research aims to generate a model of the major elements that affect the students' expectations, their experiences of pressure and their coping strategies in order to pinpoint problems and possibilities within their clinical education.

\section{Methodology}

In Grounded Theory (GT) methodology the theoretical framework is the goal, not the base, of the research (Henwood \& Pidgeon, 2003). If the researchers chooses to attempt the GT approach recommended by Glaser (e.g. 1978), as in our case, research questions are not phrased to test hypotheses but to indicate the intended direction of the study. Data, and the process of analysis, is expected to lead the investigation away from the initial questions if those are not central to the informants.

When we decided to include Connell's relational theory of gender as our analytical framework, and to pose questions about gender norms and gendered practices to all students, we deviated from Glaser's recommendation to let our questioning be guided by our data, a deviation he would have seen as "forcing the data" (Glaser, 2002). In this respect our approach is more in line with Strauss and Corbin's recommendation to make initial questions explicit and use them to direct attention to "the specific phenomena that are to be subjected to ... analytical scrutiny" (Henwood \& Pidgeon, 2003). Adding gender relations as a topic does, however, not equal posing anything like formal research questions.

As Glaser's positivist epistemology in general clashes with our (relational) gender perspective, as well as our choice to focus on experience rather than "reality," our use of the methodology and interpretation of the research process relies on the constructionist approach to GT methods applied and described by Charmaz (2003, 2006). She concludes that though the founders of GT share a (post) positivist epistemology, the tools of the method do not require researchers to adhere to the same epistemology. A constructionist approach will, however, have impact on the conclusions the researchers can make and the balance between the voices of the informants and the voice of the researcher. Consequently, we see ourselves as interpreters rather than discoverers, acknowledge that our data is socially constructed rather than objective facts, and recognize that it consists of reconstructed experiences rather than the experiences themselves (Charmaz, 2003, 2006). In the balance between giving voice to our informants and 
striving for an analytically abstracted model we find ourselves half-way between Glaser's and Charmaz's approaches.

GT methodologies as described by Glaser (1978) prescribe few, broad, and open interview questions, a flexible interviewer who follows up on subjects that appear to be central to the interviewee. This approach calls for flexible interview guides, which are expanded to include subjects introduced by interviewees as new questions in subsequent interviews, and the exclusion of themes that appear to be of little interest to the interviewees. These guidelines rule out the use of structured interviews, defined by fixed questions in a fixed order, and instead speak in favour of semi-structured interviews. In the context of this study it means that the interviewer loosely followed a thematic interview guide, flexible with the exception of the focus on the students experience and the deliberate inclusion of gender-related questions. It should, however, be noted that these gender-related questions did not remain altogether unchanged but were to a great extent adjusted according to new data.

Consequently this study consists of semi-structured interviews with twelve dental students (seven women and five men) in the eighth to tenth semester $(\mathrm{P} \approx 130$ in 2007) and three teachers at the Umeå dentistry programme (two women and one man). Except for one male 40+ outlier the students were between 24 and 28 years of age. All the interviews were conducted and analysed by the first author and the results were subsequently discussed with the co-authors, as well as a designated method specialist.

As Cutcliffe (2000) recommends that researchers begin their research by finding informants who possess the knowledge to provide a general overview of the topic, we initiated our research by contacting one male and one female experienced teacher at the dentistry programme at Umeå University. These interviews should not be seen as an inclusion of the teacher perspective in this study but as a way to gain general knowledge about the dental education and the Umeå dentistry programme. The male teacher had twenty years of teaching experience and taught theoretical subjects, whereas the female teacher had approximately eight years of experience as a clinical instructor. We also conducted an additional interview with a female teacher who had knowledge that was relevant to our research purpose. The nature of this knowledge cannot be specified due to anonymity issues.

The interviews with the teachers contained seven open-ended questions in order to produce a background to the study and to facilitate the introduction 
of additional questions based on the teachers' knowledge of the dentistry programme. The teachers were also asked to reflect on changes in the programme and the student population over the years. T wo interconnected results from our analysis of the initial teacher interviews stood out, firstly, that the dentistry programme was seen as tough and demanding, and, secondly, that our informants were confident that today's average student could meet, cope with and even exceed their expectations.

The teachers also presented dentistry as a gender-neutral profession with gendered specialisations, and possibly gendered elements. That is, dentistry in general was described as "gender neutral," which would indicate that the profession on one hand has lost its historically male connotations and that, on the other hand, the increasing amount of women as dental practitioners and students have not given dentistry unambiguous female connotations. At the same time some career paths, such as becoming a professor or an oral surgeon, were described as (still) more common or traditional choices for men. One of the teachers speculated on the possibility of describing dentistry not as gender neutral per se, but as a combination of care or caring and "things such as biomechanics," which we be interpret as an indication that different elements of dentistry can be connected to the traditional spheres of men and women respectively. These results, corroborated by student informants, led to the subsequent adjustment of our questions concerning gender and dentistry.

The tenth-semester dental students were then approached with an invitation to take part in the study, and seven female students agreed to participate. Complementary interviews were conducted with five male students of the eighth and ninth semesters the following spring. The initial interviews centred on ambition and expectations, stress, coping strategies, self-efficacy, as well as the perceived occurrence of gendered expectations and unequal treatment. A number of questions, especially questions that led to interviewee retrospection or speculation, were subsequently excluded from the study as the students found them hard to answer.

Initially we had assumed that the students of the tenth semester would be more concerned with their impending debut as fully fledged dental practitioners than the education they were soon to complete, and that the focus of their narratives would differ from that of the students from semesters eight and nine. However, this was not the case. The narratives of the tenth semester students were quite vivid in their reports on what it is to be a dental student, and the narratives of the eighth and ninth semester 
students offered reflections. We conclude that our material has not suffered in any considerable way from the fact that the female students were closer to their final exams than the male students were. Consequently, the focus of the interviews entirely shifted to the here and now of the dental programme, and included the students' worries, experience of control and their ways of coping with an environment that they perceived they had no control over.

All interviews were tape-recorded, professionally transcribed word by word, and lasted $50 \pm 20$ minutes. We decided to do the coding passage by passage, rather than the prescribed line by line, as the material consisted of many fragmented sentences and an isolated line often would make no sense at all. In all three stages of coding (open, selective, and theoretical) we strived to adhere to Charmaz (2006) practice of keeping the codes active in and representing actions in the data in order not to introduce theoretical concepts prematurely.

During the coding phases our analytic framework, Connell's relational theory of gender, was used as a sensitizing concept, that is, as a prism or lens. Charmaz describes the use of sensitizing concepts as offering "ways of seeing, organizing, and understanding experience ... [we] may use ... only as points of departure from which to study the data” (Charmaz, 2003, emphasis in original).

In the open coding phase codes were usually phrased closely to the informants' words, for example "women get more support," "trying to adjust to teachers demands," and "taking it personally." In the process of constant comparison, where previously analysed interviews are re-analysed and partially re-coded in the light of new data (Charmaz, 2006), we clumped similar codes together in order to keep the number of codes manageable, somewhat increasing the level of abstraction. For example, the codes everybody doesn't have what it takes, lazy/not good students, you react to repeated blunders and you wonder if they are in the right place together formed the category those who aren't quite up to measure.

Central to the early stage of the selective phase were lists of code counts, which served as a tool in the process of clumping similar codes together, and selecting the central codes while dropping the peripheral ones. By the end of this stage codes in general had become decidedly more abstract, for example "providing support and freedom," "prioritizing and structuring," and "to manage/keeping up." In parallel to this process, our interview guide was adjusted to data and parts of the interviews were aimed at filling in gaps, and find statements that either corroborated or contradicted previous statements. 
Eventually the analysis arrived at a stage where we had an abundance of codes and memos suggesting possible connections and categories but no obvious structure. Resolving to a paper and scissors approach, the first author cut the printed code-list so that each code ended up on a separate paper-slip. She then sprayed each slip with post-it glue to facilitate easy attachment and removal, and alternated between grouping codes that fitted together thematically and sorting codes into memo-based preliminary categories or central topics on large sheets of paper. The outcome of this selective process was several sheets of papers each representing a preliminary category. An example of the internal structure of the categories can be found in Table 1 .

Insert Table 1 about here

The relations between categories and subcategories were tracked and drawn as a category mind-map, a process that also resulted in further unification of some of the preliminary categories. It soon became apparent that the categories Assessing your own performance, Passing through the eye of the needle and Experiencing pressure and stress shared two common denominators: problems related to the students' knowledge of whether or not they have met demands or whether they have put in enough effort. Consequently Experiencing ambiguity was suggested as a core category based on the What's enough/When's enough dilemmas. The gendered dimension and the dimension of unequal treatment, which blur the solution to the dilemmas, further define the core category.

The gendered dimension originated from the open coding of informants' answers to questions that sprung from our analytical frame of gender-power relations (Connell, 2002). These codes formed a separate category for some period, but during the theoretical phase of the coding it became apparent that the model would benefit greatly if they were treated as a dimension rather than being extracted and separated. The dimension of unequal treatment underwent a similar transformation for very much the same reason. At the end of this process, and well within the theoretical coding phase, the names of the codes had not changed very much. However, what each code represented, and how the codes differed from each other as well as their interrelations had become considerably clearer. By this time it was also clear 
that many codes belonged to the Process and Strategy families respectively (Glaser, 1978).

Our tentative model was presented to two additional students, our theoretical sample, and they were asked about their opinions and impressions. The two students acknowledged that the model described their educational situation and recognised their own experiences and feelings of ambiguity. Some final adjustments were done to further refine the codes and categories, which concluded the theoretical coding, and our model was given it's (this far) final form. Though we had by now selected three additional theories to use as sensitizing concepts, presented below in the discussion, the structure of the model remained influenced by our contextual interpretation of the data and cannot be said to contain any distinct elements of other models or theories. The proposed model contains 44 codes, primarily gathered in the three categories mentioned above and the four subcategories described in the presentation of our results.

\section{Results}

We propose a model that consists of the core category Experiencing ambiguity and the three categories Experiencing pressure and stress, Assessing your own performance, and Passing through the eye of the needle. The model also contains four subcategories: Managing your time/Catching up, To manage/Standing it, Experiencing inner and outer demands and Going through the mill. Below we will present the model top-down, from core category via categories to subcategories. We will illustrate the model with typical as well as extreme statements.

Insert Figure 1. about here

\subsection{Experiencing ambiguity}

The core category Experiencing ambiguity is an attempt to capture the sense of two student-centred dilemmas that our analysis shows were present in all categories: knowing when you have achieved enough, What's enough, and knowing when you have put in enough effort, When's enough. One possible source for these dilemmas is the elevation of informal demands due to "better students." As one of the teachers put it, 
We know the grades they were admitted on so ... I even said it to them, that I have great expectations of them. --- I am prepared to argue that I cannot really burden them with too much to [comprehend], not in the way that they would be unable to grasp it ... However, they can be given ... [a too heavy workload]; you can give them too many exams and too much to study and stuff like that. But then again, if you compare it to the dip we had in the '80s-' 90 s, in those days you really didn't know what you could ask of the students, because ... I simply didn't think they could handle it.

From the students' point of view, however, it did not seem as if they needed less support but support of a different kind. They expressed the need for help in prioritising and managing their time and resources, that is, solving the two dilemmas. The students' ability to prioritise seems to contain an element of maturity and life experience; it was something you either had with you when you started the programme or had to learn, as one student put it, "terribly fast." If not before, the shift away from what one student called "the upper secondary school mentality" came in the clinical semesters. As a female student put it:

\footnotetext{
But for me it was just, well, from one school to the other and it became like - you were thinking along the same lines as when you went to school and then it became apparent that 'Hey, I'm actually going to work with this. It's like, it's people I am going to work on and all that, and I have to know this.' ... That was a tough changeover ...
}

Students seem uncertain about how much work they are actually required to do. Ambitious students seem to see no limit to the effort they can or have to put into the programme, whereas their peers who have other priorities pass seemingly without effort. One female student put it like this:

Many would probably say that it has been stressful ... and that it has been very hard work, and a tough education, and intense. But there are probably some that would say that, no it has not been stressful. Because they haven't attended lectures .... and they haven't attended the clinic.

Students attempt to solve the When's enough/What's enough dilemmas, and succeed to a greater or lesser degree. How they reason when they prioritise their time and effort is central to the subcategory Managing your time/catching up. Some see it as a matter of structuring and planning, others 
see no other way than to wear themselves out. "It's just to shift into top gear and drive "til the gearbox falls off" one female student put it. How students relate to the upper as well as the lower limits of their expected performance is captured mainly in the subcategory Experiencing inner and outer demands, and their ways and possibilities to cope with pressure and stress is the theme of the subcategory To manage/Standing it.

An unsolved What's enough dilemma affects students' interpretations of their own effort, performance and accomplishment. The category Assessing your own performance is especially central in understanding this dilemma. Feedback is not experienced as continuous, consequent or unbiased. One female student stated that "you can feel the pressure and the demands but you don't know where you stand. --- I never really ... believe I meet those demands well."

A male student felt that previous mistakes kept influencing the teachers' assessments and felt, without being absolutely sure, that their negative assessment was unjustified. This scenario creates a conflict between the teacher's assessment and the student's self-assessment, which leads to one of the assessments being disregarded by the student.

\footnotetext{
I try to be honest with myself at all times, so this is something I really believe that I am, good that is. But when I hear, like when I heard that I'm unprofessional with patients ... I hope I know myself well enough to take that kind of critique, and not lie to myself entirely so that I actually am very unprofessional with the patients ...
}

There also seems to be a problem with different teachers treating demands differently, which makes it even harder for the students to solve the What's enough dilemma. For example, one student commented that demands on students to perform a fixed amount of procedures on patients were sometimes flexible due to the lack of patients and clinical facilities, and that newer/younger clinical instructors were more inclined to stick to the formal demands than more senior staff.

The formal or formalised answers to the What's enough dilemma, as well as the students doubts in the usefulness and substance of the formal demands, lie in the category Passing through the eye of the needle. The category is centred on the conflict between high admittance scores, and presumably unbending minimum standards, and students that are awarded the degree but are not believed to be up to standard, which implies 
negotiable minimum demands. The notion that a few students seem to "slip through the check-points" was expressed by both teachers and students, either as a possible risk or as a supposed fact. As one female student put it:

\begin{abstract}
But there are those who don't have super ... Let's say that, well, you might not go and sit down in the dentist chair of some [fellow students]. And it's somewhat frightening that they will graduate in about a week.
\end{abstract}

When's enough, on the other hand, is a dilemma connected to the toughness of the dentistry programme and the students' questioning of the origin and benefits of the toughness. As one male student put it, "you don't become a better dentist because it's tough. Not only from the 'being tough' thing ..." The traditional toughness as well as the students' reservations are caught in the subcategory Going through the mill. When's enough is also connected to the subcategory Experiencing inner and outer demands, especially in regard to the ways in which high inner demands affect the students' ambitions and efforts.

The solution to the students' When's enough/What's enough dilemmas is further obfuscated by two dimensions that are central to the core category and run as undercurrents in all categories and subcategories: the gendered dimension and the dimension of unequal treatment. The gendered dimension refers to the informants' notions of (sex) gender differences and (societal) norms, and the dimension of unequal treatment refers to notions about cultural or personal misunderstandings, misinterpretations and incompatibility in teacher-student relations. These two dimensions do in effect present students with multiple and contradictory sets of rules or roles.

Our informants explicitly related to dentistry as a gender-neutral profession. However, the gendered dimension is expressed in relation to the male dentist norms and the female student norms. One example of this is the informants' notion that male and female students are equally well suited for the profession, but might become slightly different kinds of dentist in relation to career path and/or approach. Almost all of the informants touched on the stereotyped notion of the caring woman, and female students tended to relate to this stereotype either by conforming to it, and thus presenting themselves as a different type of dentist in relation to the male norm, or by transgressing the stereotype and, for example, aspiring to specialise in oral surgery, which is a career path that is still dominated by men. 
Women and men also experienced that they were treated differently and perceived as different types of students. Female students felt that they were expected to be more orderly and hardworking, and believed that male students took things more lightly, were given more leeway and could "get away with a joke." One female student also said that she believed that "guys have a really good ability to be content with what they have achieved" and that female students tended to be less satisfied when they assessed their own performance.

Even though a few male teachers were believed by students of both sexes to give more attention and help to (young) female students, female as well as male students tended to state that their opposite sex was privileged in teacher-student relations. The teachers stated that same-sex student-teacher relations facilitated communication, and they expected male students to be more privileged in mixed relations, especially in the interaction with dental nurses.

\footnotetext{
But, sure, sometimes you see a difference, male teachers for example are nicer to female students and the opposite, female teachers are nicer to male [students]. ... We had a teacher who was, well, for example ... he was with me [supervising] two three minutes tops, and then when the girls [ask for help] ... fifteen, twenty minutes he can stand there and help them!

... and it has always been the [female] dental nurse who has cleared up and fixed [stuff] so it is sort of taken for granted that when another woman arrives she performs the same tasks. I am not against it; dentists can perform these tasks just as well as the dental nurse. I don't think that's odd. But it's somewhat sad that it's expected of girls [us female students], but if a guy does it it's considered something of a bonus.
}

Apart from being central to the core-category, the gendered dimension is also prominent in the category Assessing your own performance.

The dimension of unequal treatment contains elements of ethnical stereotyping. The problem-students who teachers and students sometimes referred to were almost exclusively male and quite often referred to as nonSwedish. In one interview a female student stated that non-Swedish male students "are expected to be somewhat worse, or not worse, you could perhaps say that they are expected to be somewhat troublesome." Another female student believed that ethnicity was less of a problem in her cohort 
than in previous cohorts, because the teachers had become more used to a heterogeneous student population.

In general, the possibility of ethnical discrimination was downplayed by students as well as by teachers and (culture and/or language related) misunderstandings, thoughtlessness and personal attributes were brought forward as alternative explanations. As a matter of fact, in some statements "personal chemistry" seemed to be considered one of the major factors behind unequal treatment, and students of Swedish descent did not believe they were excluded from the influence of "chemistry." In the statements from two male students these beliefs are quite explicit:

\footnotetext{
The higher the semester, the more dependent you become on your supervisor. ... When everything is theoretical you are anonymous. But ... now you have a rather close relation, really, to your clinical instructors and if the personal chemistry does not click ... there are many who, at least from what I've heard, get into trouble and often people say it's because of personal chemistry.
}

There is so much at stake ... There's so very much at stake, it feels as if my whole life is at stake and it could all come to nothing because of such a ... such a random thing as the personal chemistry between me and the supervisor ... if it clicks ...!

The dimension of unequal treatment is particularly well manifested in the categories Experiencing pressure and stress and Assessing your own performance.

\subsection{Experiencing pressure and stress}

The category Experiencing pressure and stress is based on our analyses of statements relating to stress, the fear of falling behind, the prioritization of school performance, or the lack of prioritising skills. It contains the subcategories Managing your time/Catching up, To manage/Standing it and Experiencing inner and outer demands. Experiencing pressure and stress is central to the What's enough and the When's enough dilemmas, and the dimension of unequal treatment is evident within the category.

The interviewed teachers had indicated that whereas female students might speak of stress-related issues the problems experienced by their male peers were primarily of a more practical nature, such as a wish to change base group. However, in the interviews male students spoke freely about 
their own stress or the stress of other male students. The stories told by male students tended to be sorted into codes that were related to unclear outer demands and the problems of knowing whether or not demands had been met. On the other hand, the stories told by female students tended to be sorted into codes that were related to coping with inner demands.

Stress and pressure were apparent but complex to capture in code. Time was a factor for the students, but so were demands that had to be met in order to be allowed to continue to the next course or level, the fear of illness and the consequences of falling behind. Other prominent issues in relation to stress and pressure were the responsibilities connected to making judgements, especially in unfamiliar situations or when there was a perceived lack of control, and being assessed. The code all that surrounds it is an attempt to capture the students' notion that demands seem reasonable on paper, but that the groundwork that has to be done in order to meet these demands, and the ease with which careful preparations may come to nothing, is a taxing experience. One student remarked that he used to be an easygoing person who never really had experienced stress and pressure before entering the dentistry programme. He felt that there was a tangible risk that he could fail due to circumstances that were outside of his control, which contributed substantially to his stress. All in all, lack of control-as captured in, for example, the code being powerless or without at choice-seems to be a major contributor to student stress. As one male student put it:

\footnotetext{
Well, first and foremost you should never be ill. I've been ill ... twice when we had clinic [practice] ... and I have felt sick from it as I immediately would fall behind everybody else. So in effect I cannot be ill.
}

Students' relation to the clinic seems to be at the crux of the matter. Practicing procedures on patients in the student driven clinic was soon established as something positive, and some students claimed that the clinic was what made their theoretical studies make sense. However, clinical practice was simultaneously seen as a source of stress and pressure because of a perceived lack of control over teacher availability and levels of support and supervision, the risk of being overruled in matters of patient treatment. As there is a stipulated amount of times a student needed to practice a procedure within a course, students also worried that their patients would not show up or would not need the kind of procedure the students were supposed to be training at the time, in which case they would have to find an 
additional patient to book. A male student described treating a patient as a possible "conflict situation":

\begin{abstract}
In the situation when you have patients and you want to perform a certain treatment, the patient might have a wish and then you have a teacher with an opinion ... it develops into something of a conflict situation after all and I think it's ... You cannot really control the situation all the time even though in effect you are the one performing the treatment.
\end{abstract}

The subcategory Managing your time/catching up is based on the students' worries about having the time to keep up or catch up when they have fallen behind, as well as their strategies, experienced alternatives and possibilities to manage their time. Insufficient time in relation to expected performance and amount of responsibility was a salient factor in student statements. The students' notion that they were expected to sacrifice their spare time, weekends and even holidays to manage their schoolwork was supported by the teachers who, among other things, stated that:

\footnotetext{
It's not like other [university-programmes] ... where you have certain lectures and then you are free to go, that is not the case here since [so much of the clinical education] is tied to the [student driven] clinic. --- I believe that if they compare themselves with other [students], they have a lot of scheduled time. The studying comes on top of that in the evenings and at the weekends, and I believe they think that is tough.
}

The skill to structure one's studies, plan one's time and make realistic priorities was seen as a stress reducing factor by students and teachers alike, and was associated with maturity, perspective and (working-)life experience. Two factors that the interviewees related to prioritisation were ambition and attendance. The range of student statements span from the considerable amount of time a student could spend with programme-related work that was driven by ambition, via how much time that should be spent in order to keep up standards and meet demands to how little time some students were suspected to spend on theoretical studies and clinical practice and yet not be cut off from programme or have to repeat a course.

The code prioritising/structuring your time, though highly relevant to Managing your time/catching up is perhaps more central to the subcategory To manage/Standing it. This subcategory is an attempt to capture the students' stress-management strategies, coping resources and their ways of 
coping with their experiences of stress and pressure. One student claimed that he would rather miss an examination and have time to study for the reexamination than have to endure the stress involved in last-minute revision. The reason he stated for this strategy was that he had learned to prioritise his well-being. He would also rather accept a lower grade than place himself under severe stress, and felt confident that he was good enough to become a dentist anyway. However, the opposite strategy seemed to be more commonly employed. In the words of a male student:

\footnotetext{
[A class-mate who entered the dentistry programme directly after finishing upper secondary school], he studied so much that he became ill ... and he hardly slept at night and he was very tired in the day. ... [And] there are girls who are fresh out of upper secondary school and who, how shall I put it, they want to achieve so much ... they want to be best at everything ... They have so many demands to live up to.
}

According to a number of students, the culture of the dentistry programme promotes the idea that you should hide your stress, you should just stand it and keep your tears and complaints to yourself. Complaining and asking for support from a teacher was considered to be problematic by some students, as the same teacher would also be responsible for performance assessments, and they called for another solution. A female student wished for "someone to turn to who isn't the director of studies or senior faculty members who also ... assesses your performance and decides your grades, because it can like clash, but still [a person who] ... has insight into the whole situation and knows how tough it can be."

Students gave a full spectrum of answers from positive to negative in relation to whether or not complaining would change anything for the better. Some students stated that they went against the programme culture and showed emotions, expressed stress, protested and asked for support from peers and teachers. One female student concluded that "you feel as if it's like a weakness if you show emotions in front of a teacher ... But it would be good to show them because they would perhaps learn something from it.”

As mentioned earlier, clinic duty was also experienced as means of coping as it helped to make the theoretical studies relevant. To successfully treat a patient was experienced as rewarding and turning knowledge into practice could increase students' self-efficacy.

The students were also explicitly asked whether, and in what way, they experienced inner and outer demands, and their answers were gathered 
chiefly in the subcategory Experiencing inner and outer demands. All the female students related their own experiences to both types of demands, and the code experiencing inner demands soon included statements about gender norms, coping, wellbeing and altered experience; school seemed tougher for those with high inner demands because they also experienced the outer demands to be higher. One female student said that “... if you are someone who already has high demands on yourself, and the school then adds really high demands, the demands become enormous and, well, they form like a perpetual upwards spiral." Some, but not all, of the male students talked about experiencing inner demands. Perhaps the best example of a male student referring chiefly to outer demands is the student who stated that: "My demands ... are secondary. Instead it is first and foremost I who shall fulfil ... the teachers demands."

During our analysis we noticed that the students' belief in the relevance, rigidity and educational value of the demands seemed to be important dimensions of the code experiencing outer demands.

\footnotetext{
It would seem that the requirements are quite flexible. But I think it's because the teachers realise that they cannot insist on the preset minimum requirements ... because there's a lack of time and a lack of patients.
}

Even though most students experienced outer demands as somewhat unclear and fluid, there are statements in which students express confidence in the strictness of the demands at the dentistry programme. As one male student states, "We all feel different subjective demands, but there are in fact stipulated demands as to what a dental student should learn and know."

\subsection{Assessing your own performance}

The category Assessing your own performance is based on our analysis of students' statements regarding experienced (lack of) feedback, studentperformance comparisons, and the degree of insight that they experienced in relation to their own (clinical) performances as well as the degree of insight they believed was experienced by their peers. This category is central in understanding the What's enough dilemma. Female students stated that they believed that their self-assessment and the way they were assessed by others were based on a stricter standard than that used for the assessment of, and self-assessment by, their male peers. Consequently, the gendered dimension is prominent in this category. 
In general students said that they were pretty sure that they would be informed when they did something wrong, but that positive feedback was comparatively rare. Students wished for more feedback as well as more continuous feedback on their clinical performance and complained that despite feedback being scheduled, there was seldom time for it. It did however seem to be possible for students to actively demand feedback. One female student concludes that "the students who talk are the students that get feedback," a notion that was later corroborated by the only student who was content with teacher feedback.

Both teachers and students concluded that a small number of students seemed to be unaware of or refused to acknowledge their shortcomings. Students explained this phenomenon by referring to personality traits or culturally related attitudes, and expressed worries or frustration about the lack of feedback from teachers or other actions to regulate or stop these students. One student vented her frustration by saying that "it feels as if some students just slip by ... [I wonder if] they even talked with a clinical instructor and received feedback.” Teachers also expressed concern about students with this attitude or trait and talked about communication problems that they believed often were enhanced by cultural differences. As one teacher expressed it:

\footnotetext{
In Sweden I would say we have a tendency to systematically understate our own performance, knowing very well that everyone knows that we are better than we say we are. ... I have experience of the American culture [USA] which is completely different in that regard.
}

The category Assessing your own performance also contains the subcategory Experiencing inner and outer demands. In part this relation has to do with the way in which students' inner demands relate to their selfassessment, and in part it is due to the problems that they expressed in relating performance to unclear outer demands. As mentioned above, the relation to inner demands was more marked in the statements of female students. The frustration regarding unclear outer demands was felt by male and female students alike, but most of the statements coded as relating to outer demands only were made by male students.

Students felt that they had to deal with contradicting information and assessments from different teachers. Sometimes what teachers told them would go against what they themselves believed to be best, correct or in 
relation to their actual performance. One student commented that he had found these contradicting messages stressful at first, but that "you form your own picture, you create your own reality instead of buying in on someone else's." Another student said that it had taken him a while, but he had come to believe that the teachers relied on what students told them they could do rather than on forming an opinion by observing them and asking questions. His conclusion was that the teachers therefore might believe that quiet students were less proficient or knowledgeable.

\subsection{Passing through the eye of the needle}

The third and final category, Passing through the eye of the needle, stems from our analysis of codes related to high admittance scores, high demands during the course of the programme and from concerns about these demands not guaranteeing the quality of neither the education nor of the dentists who passed their university degree. These conflicting notions position the What's enough dilemma firmly within the most formal and formalised demands of the Umeå dentist programme.

One student believed that it was impossible to stop all the students who were not fit to become good dentists by enforcing rules and demands, but that unnecessary strictness eliminated some of the students "fit to be dentists.” This notion was also expressed by other students in, for example, this rhetorical statement made by a male student: "[Do you mean the] admittance requirements or [the requirements for passing courses] ... or both? Well, the requirements for admittance, they are really strict. Over time I have become a little more disillusioned [about the actual requirements for the minimum passing grade].”

Passing through the eye of the needle also contains the subcategory Going through the mill. This subcategory rests on our analysis of codes that were related to notions about the Umeå dentistry programme as a tough programme, and the students' views on the origins, necessities and benefits of this toughness.

Students and teachers alike talked about the dentistry programme as one of the tougher, or even toughest, programmes at Umeå University: it has a tradition of being tough, and maintains that tradition. In the words of one female teacher and one female student:

Well, during the period I have them [as students] they don't speak to me about it being unreasonable. It's more that some 
..., but it afflicts them all really, it's more like a culture, that this is the way it should be.

The teachers say that ... or actually it's mostly the lecturers that say [things like], 'Well, you should know that this is among the toughest educations, and it's cool that you attend' or some such thing ...

The gendered dimension is present in this subcategory since the toughness and the way it has become a part of the programme culture parallels a traditional male (medical) hierarchy (e.g. Davies, 2001), and contains elements similar to a rite of passage. Two female students put it like this:

\begin{abstract}
Because if you have completed this tough an education ... well, then you are quite accomplished so then, then it's just to take your experience(s) and go for it, and not give up when things go awry. Really. Because it is an accomplishment, I believe, to have made it.
\end{abstract}

Sometimes you feel like, yes it's tough right now, but we have all sweated it out, it's like [the teachers tell you that] 'Yes, we know you have a really tough time right now but that's the way it is.' ... It's not that pronounced [among students] but it's like, well, you deal with it, it's tough but you just see it through.

In especially the analyses of the interviews with the female students, codes regarding inner and outer demands tended to be tagged to statements which had also been assigned the codes programme culture and/or to maintain a stiff upper lip.

\begin{abstract}
Well, there's this jargon that as a student you're not worth very much, and ... though it doesn't show on paper, there's a rather strict hierarchy. And ... you cannot muster the effort to do something about it even if you believe that something's not right, or if you believe something should be changed, because ... you'd have a hard time getting your voice heard.
\end{abstract}

Students agreed that much of the toughness could be reduced through organisational improvements, coordination and a thorough overhaul of the dentistry programme's courses and schedules. In multiple statements students claimed that these shortcomings had been brought up by themselves or other students but that little has changed. According to one female 
student, alumni who had attended the Umeå dentistry programme in the 1980s had recently commented that current schedules seemed to contain the same problems they remembered from their own education, and that they recognised complaints that seemed not to have been fixed in twenty years.

No one claimed that dental students had to be smarter than the average person to pass the dentistry programme, but our informants did claim that the programme was strenuous, focused on performance, and that it was quite normal for students to feel considerable amounts of stress and pressure. More than one student statement was coded as you should always be at your peak [performance].

\section{Discussion}

The proposed model consists of the core category Experiencing ambiguity and the three categories Experiencing pressure and stress, Assessing your own performance, and Passing through the eye of the needle as well as the four subcategories Managing your time/Catching up, To manage/Standing it, Experiencing inner and outer demands, and Going through the mill.

At the core of our findings lies ambiguity, which was captured in the student dilemmas What's enough/When's enough. The answers to these dilemmas are further complicated by the gendered dimension and the dimension of unequal treatment that provide students with different and contradictory sets of rules and roles. From a gender perspective it is possible to argue that female and male students attend parallel programmes and learn to adhere to a different set of gendered rules/roles in order to enter a profession that our informants refer to as gender neutral.

This study indicates that the Umeå dentistry programme is taxing on the students' time and (inner) resources. The informants report that the dentistry programme is considered to be tough, but seem to relate this toughness to the culture (mainly attitudes and traditions), and context (mainly conditions and resources) that surround the curriculum rather than what it takes to become a dentist. The students' experiences of their education are also highly influenced by the way they relate to the culture and context of their education; both of which may be either elusive, hard to interpret, hard to control, or all of the above. It should also be noted that our results indicate that the combination of being a woman and fresh from upper secondary school, the single largest demographic student group at the Umeå dentistry programme, may increase the amount of stress and pressure perceived by these students. 
However, the situation at the Umeå dentistry programme is not unique. A recent study from the U.S. concluded that

\begin{abstract}
dental students viewed their clinical education as being a positive experience with four notable exceptions that students perceived to be largely out of their control: 1) limited numbers and accessibility of faculty, 2) inconsistent and all too often inconsiderate feedback by faculty, 3) clinic inefficiency and lack of support resources, which required students to perform administrative tasks that detracted from opportunities to learn, and 4) strategies required to meet procedural requirements that were described by some students as being ethically questionable (Henzi et al., 2006, pp. 375-376).
\end{abstract}

The results from Henzi et al.'s follow-up study (2007), which was a SWOT analysis based on students' perception, also supports our results. For example, dental students identified disorganisation —-both in clinical learning and the overall curriculum — and the clinical instructors' inconsistent student assessment as the major weaknesses of their education.

Below we will discuss central aspects of the Experiencing ambiguitymodel in relation to Connells' relational theory of gender, which has served as our analytic frame throughout the study. We will also introduce three additional theoretical concepts and models: the demand-control-support model and feedback in relation to the concept of self-efficacy. By introducing these additional analytical frames we intend to achieve two goals: to position our model within the realms of work and organisational as well as social psychology and to introduce tools that will strengthen the validity and reliability of our follow-up studies.

\title{
4.1 Ambiguity, blurring and gender-power relations
}

Both the gendered dimension and the dimension of unequal treatment can be interpreted from power perspectives, the former from a gender-power perspective and the latter from the power imbalance inherent in the teacherstudent relation.

First we focus on the students' belief that the faculty members and staff practice different rules and apply these rules differently based on the students' sex. As the clinical semesters of the dentistry programme contains both educational and medical (hierarchical) contexts, our analysis will consider both structures. Gender norms in the context of education seem to take female students' high achievement levels and good behaviour for 
granted, which is a phenomenon that is frequently observed in educational research (e.g. Jones \& Myhill, 2004). Such behaviour is generally rewarded with good grades. Our results indicate that previously successful, though probably not un-stressful, strategies may leave students with problems concerning prioritisation in the clinical semesters. As our informants believed that this problem was best solved through (working-)life experience and less focus on academic achievement, the behaviour of "ideal" female students may be regarded as immature in this context. It is possible that the expectations that the female students believe that the faculty members have of their performance prevents them from maturing/prioritising to some degree and thereby exerts power over their progress in the clinical semesters.

Research on medical settings (e.g. Eriksson, 2003) suggest that becoming a medical professional includes showing your mettle or going through the mill, which can be associated with rites of passage (formerly) common in male hierarchies (Davies, 2001). It seems as the culture of the dentistry programme still encompasses the notion of the programme as something tough that every dentist has gone through, and that every dental student should go through. This notion exists even though the benefit of this "rite of toughness" is questioned by some or most of today's students, male as well as female. If going through the mill does not lead to pride or privileges, the rite will inevitably lose its relevance. It would therefore be interesting to probe which qualities of the programme further the feeling of accomplishment, pride and content and how this relates to cultural background, gender and the perceived effort of one's peers.

Within the medical/clinical context we also believe that the nurse-student relation is interesting from a power perspective. According to our informants, dental nurses assisted male students more willingly and showed a greater degree of acceptance when they were less orderly than they did with female students. This pattern can be found in other medical settings (Cassell, 1997; Davies, 2001; Eriksson, 2003), which implies that there is a half step, between nurse and dentist, on the hierarchical ladder that is only for women. It is possible to argue that members of the staff exercise their power to keep female students in a subordinate position where they are expected to be rule obeying and facilitate or even perform the nurses' tasks, while male students are allowed to disobey rules, increase nurses' workburden and receive the assistance traditionally given to male dentists.

The informants' main explanation for gender differences in relation to rules was that they reflected societal gender norms. As a consequence, 
resistance becomes harder since this notion implies that it is the societal order and not the programme that wields the power over the gender relations and therefore should be the target for resistance. Since this power is also chiefly discursive, resistance becomes harder but not impossible (Connell, 2002). A start would be if the faculty, staff and students began to view themselves as actors doing gender in their own context (e.g. West \& Zimmerman, 1987) instead of performing as socio-cultural re-producers of gendered norms (e.g. Bussey \& Bandura, 1999).

The "rule and role" differences described by our informants can also be analysed along the dimension of production relations (Connell, 2002). Gamarnikow (1978) draws a parallel between the nurse-doctor-patient triad and the family structure: nurturing mother/nurse, patriarchal father/doctor, and dependent child/patient. According to Davies (2001), this patriarchal structure is changing with the norm of patient-centred care and as nursing has become more of a profession. This has led to a change in the the doctor/nurse relation, and the hierarchical/structural differences between these two profession has become less pronounced. These trends, which are paralleled in dentistry, strengthen the positions of the nurse and patient respectively. In her own research Davies found that while male doctors are still expected to have and execute authority vis-à-vis nurses and patients, female doctors who exhibit the same behaviour may be frowned upon, which further changes the interaction within the triad. These changes may lead to a new kind of medical doctor, who relies more on relational skills than authority, and Davies states that "[It] is often in this intersection of caring and curing that the possible (and assumed) contribution of women doctors is raised - frequently in relation to a more essentialist gendered understanding” (Davies, 2001 p. 31).

We can conclude that the notion of caring as the hallmark of a stereotyped female dentist appears in our results, and that statistics imply that female dentists may outnumber their male colleagues in the foreseeable future (Nunn et al., 2008). It is quite possible that, for good or bad, women will soon be the dentist norm and their male colleagues will no longer "get away with" for example being authoritarian or leave for the nurse to clean up the workplace. However, to assume that feminisation by number will lead to a more feminine ideal physician or dentist would come too close to the presumption that there is an inherent feminine nature.

Two parallel questions that arise are: what happens when women wear the white dentist coat, and what happens to the white coat when it is worn by 
women. These questions are relevant since all Umeå dental students wear white coats in their clinical semesters. The white coat is a major symbol of (male) medical authority (Connell, 2002), and female doctors who wear it bear witness that (older) patients ask them when the doctor will come (Eriksson, 2003). As previously discussed, female doctors/dentists are not expected to execute the kind of authority associated with their male peers and the symbolic white coat (Davies, 2001). Even today, it would seem that the white coat is not an unambiguous symbol of a doctor/dentist without a male face to go with it.

The traditional "white coat romance" (as portrayed in fiction) between male doctor and female nurse has, for practical reasons, become an object of the past, but the emotional gender-power relation (Connell, 2002) seems to be a factor at the Umeå dentistry programme. It is implied by students' statements that male faculty members were, at times, more attentive and helpful towards young female students, and that female faculty members and staff were more forgiving and helpful towards male students. In the latter case, our results do not inform us whether male students have an advantage from being the minority, i.e. because they stand out, or whether the privileges involve gender norms or emotional relations. However, results presented by Freeman et al. (2007) indicate that flirting might sometimes give male students an advantage in nurse-student relations.

All in all, it seems safe to conclude that the hetero-romantic norm decides to some extent how attentive and helpful faculty members and/or staff will be towards students. We argue that even if these examples seem innocent enough, they are problematic in two ways: those students who adhere more closely to the romantic ideals of those higher up the hierarchical ladder may benefit at the expense of their less "attractive" peers. If help and attention are reserved rather that equally distributed, future services may be accompanied by implicit or explicit preconditions. It should be noted that none of our student informants spoke of sexual harassment at any time during the interviews, but, according to statistics, seven percent of all Swedish female medical/dental/pharmaceutical students have experienced sexual harassment from a faculty member (Studentspegeln, 2007). Among their male peers a total of about 2 percent reported that they had experienced sexual harassment, but the report does not specify if from faculty, staff or fellow student. 


\section{Students experienced lack of power}

Though the demand-control-support model (Karasek \& Theorell, 1990) originally focused on workers, two recent studies have shown that the model can also be successfully used to analyse students' experiences of higher education (Cotton, Dollard \& de Jonge, 2002; Chambel \& Curral, 2005). The results of both studies suggest that high demands, low levels of control and low levels of support from student peers affects students' well-being negatively.

Explicit demands as such have not been central in this study, but rather the rigidity or flexibility of the demands and the students' lack of control over their chances in meeting those demands. For example, the demands that a student must perform a procedure a certain amount of times created concern among the students. There was always the risk that the patients would not be available or not turn up at the appointed time, and these eventualities were beyond their control. Instructors might lower demands for practical reasons but, according to students, these decisions were all too often influenced by good or bad student-teacher relations. The teacherstudent power relation is central to the clinical education within the dentistry programme, and it is in this relation that the students stressed their own low status and the importance of "personal chemistry." As far as we can discern, students use the concept of "personal chemistry" to express that their interpersonal relations with, for example their clinical instructors, are crucial to the way that they are assessed and the respective levels of their grades.

This power-relation seems closely connected to control as it is expressed in the demand-control-support model (Karasek \& Theorell, 1990). However, it should also be noted that the hierarchy that is expressed by the student informants mirrors a traditional medical hierarchy that stems from the days when doctors were men, students were aspiring male apprentices and women were nurses (Davies, 2001). As students stated that they stand on the lowest rung of the hierarchical ladder, it is possible that they use the notion of "personal chemistry" as a way of making unequal application of rules or discrimination personal rather than structural, random rather than systematic. It is possible that the male/medical hierarchies not only leave the students with little or no control, but that they may also be treated differently if they conform to or transgress the norms of their sex or their chosen profession. This position may well lead to a scenario in which female students are the underdogs twice over. 
Even though clinical supervisors may lower demands for practical reasons, it seems as if there is a tendency that expectations, and consequently demands on students, are on the increase as the required admittance scores become higher. However, these new demands are not established in the curriculum and remain largely undefined and thus unattainable for the students. We argue that the dentistry programme would benefit from a formalisation of these demands, both in terms of student evaluations and feelings of control over their situation. As our results suggest that teachers think that today's students can be given more responsibilities and that they can meet demands better than students in the past, attention has to been drawn to the fact that other considerations have to be made. We believe that dental programmes need to establish ways of informing students that they have done "enough" as there are students who see no upper limit to the (inner) demands they should meet.

Our results indicate that the students' greatest control issue was the fear of falling irreparably behind due to illness and not being allowed to continue their studies. Apart from catching up on their studies during holidays, or hoping that there would be a place for them in the programme the following year-which is only guaranteed if they return from a registered leave of sickness (Umeå dentistry programme - study programme, 2006) - the students seemed unaware of other possible solutions if they fell behind. The fear of failing critical examinations was also tangible as students are not allowed to continue with the next course or clinical procedure if they fail certain tests. The fact that the teacher informants presented somewhat more flexible solutions, which were well within the boundaries of the established supportive measures, implies that there is a deficit in the communication between faculty/staff and students. This failure of communication could be caused by faculty and staff underestimating the students' fears of falling behind in their studies.

Though it was female students who stated that it was part of the programme culture to maintain a façade in school and not to complain to teachers (or peers), it is a cultural element that has male connotations (Courtenay, 2000) and it would affect all students who conform to the norm or who interact with conforming peers. This description of the programme culture suggests that social support in the students' school environment is scarce, and that stress and pressure have to be dealt with either in private or outside the context of the dentistry programme. According to the demandcontrol-support model, a programme culture that does not facilitate social 
support does little to ease the pressure and stress that is experienced by students (Karasek \& Theorell, 1990). We believe that the programme culture needs to be subjected to further studies, and that its consequences for students' well-being need to be examined.

We also observe what seems to be a contradiction between the notion that male students experience less stress, which was expressed by the female students and our teacher informants, and the high stress levels expressed by most male students in our study. It is probable that the "see it through"attitude prescribed by the programme-culture, in combination with dominating societal norms for constructing hegemonic masculinities that prohibit men from expressing their stress (Courtenay, 2000), leads to a scenario in which stress among male students is both under-reported and underestimated. These societal norms may also make male students especially reluctant to seek social support, which may indirectly increase their stress (Karasek \& Theorell, 1990).

Our results suggest that gender/sex and (working) life experience relate to the way that stress is experienced, communicated, and the way that students describe their coping strategies, and we believe that this angle should be further explored. The students' lack of control in finding, booking and treating the necessary number of patients who have the required needs, is a problem that was also observed by Henzi et al. (2006). A change in the routines surrounding these matters may well have a considerably positive impact on student stress. What else lies behind students' experienced lack of control needs to be further probed and defined.

\section{Self-assessment and feedback}

The teachers who participated in our study mentioned that there were students who were biased towards the positive in their self-appraisals. They believed these biases could be explained by culturally or personality related arrogance, or individual judgemental errors. However, as implied by our results, students have to deal with contradictory feedback from different sources, which inevitably leads to a situation in which the student disregards parts of the feedback. Over time, more and more feedback may be ignored, which would result in the development of a lack of respect for the opinion of a number of the teachers (Hattie \& Timperley, 2007). Solely negative feedback from teachers may be disregarded by students who believed that they had successfully carried out the clinical procedures criticised. 
The students in our study questioned the value of teacher feedback. Unspecific, understated, or delayed feedback will not provide a student with enough constructive pointers to change specific behaviours (Hattie \& Timperley, 2007). Moreover, feedback is affected by the learner's selfefficacy and at the same time an element that influences the forming of selfefficacy beliefs. Feedback that leaves the assessment up to the teacher and does not support self-regulation is less likely to help students build wellgrounded self-efficacy beliefs or lead to realistic self-assessment (Bandura, 1997; Hattie \& Timperley, 2007).

It is also possible that some students feel that they can rule out negative feedback on the grounds of misunderstandings in relation to how they told their story rather than their actual actions. Actual or perceived contradictions in how teachers appraise different students or student groups may also lead students to disregard critique on the grounds that it is biased. This sifting of information corresponds to Hattie and Timperley's notion of feedback as "information about the content and/or understanding of the constructions that students have made from their learning experience" (Hattie \& Timperley, 2007, p 82). Feedback is not simply direct input to further action but can be accepted, modified or rejected.

In summary, students' dissatisfaction with teacher feedback as well as self-assessment biases may reflect their difficulties in understanding the nature of the feedback they are given, and their problems to relate feedback to specific actions. It is important to further investigate to what extent students believe that their teachers can assess their actual performance, what feedback students tend to disregard, and on what grounds. In this future research all four levels of Hattie and Timperley's model should be included: the task, the process, self-regulation and the self (Hattie \& Timperley, 2007).

\section{The significance of our findings}

We believe that our choice and use of Grounded Theory (GT) methodology has facilitated credible results, and that our theoretical frames have provided valuable bases for analysis. We prefer not to speculate about the extent to which these results are transferable to related contexts, but the similarities with the results of studies by Henzi et al. $(2005,2006,2007)$ seem to vouch for the applicability of our model outside the narrow context of the Umeå dentist programme. In our future quantitative research we plan to test the Experiencing Ambiguity-model on a larger sample of dental students and to use instruments that are related to the established models discussed above 
and as well as instruments based on elements of the proposed model that is the result of this study.

As data collection and analyses were not always carried out simultaneously, salient concepts may have been favoured at the cost of implicit ones, which can limit the scope of our model. On the other hand, we have purposefully expanded the model to include a gender perspective. This line of questioning was brought up in all the interviews regardless of the informants' interest. This approach is not orthodox GT, and would not be approved by Glaser (e.g. 2002) because these questions derive from the researchers' interest. Even though our use of GT is chiefly inspired by Glaser (1978), we use GT within a feminist social constructivist framework that is influenced by the work of Charmaz (e.g. Charmaz, 2003, 2006). Our approach has also led to a greater focus on the informants' stories, which is a solution favoured by Charmaz (2006) but not by Glaser (2002). We argue that the gender perspective has proved relevant to this study, and that the model that was constructed by the interaction between researcher and informants fits, works and is valid within its context.

The gender perspective has also shown itself to be slightly problematic. Firstly, we have chosen to use to the concepts of 'female' and 'male' in relation to students and teachers because of linguistic reasons, though we are aware of the biological connotation of these concepts. Secondly, the medical context makes it problematic to present our informants' notions of caring women, and a possible future caring dentist norm, because caring/care giving is firmly established as the domain of nursing and nursing research. Thirdly, female students tended to relate their adherence to or their transgresssion of the dentist norms to their own degree of femininity, thus including femininity in their discussion of the female dentist. These theoretical troubles need to be addressed and further explored in our future research.

The results of the present study constitute a platform for future research on how dental students experience the clinical part of the dentistry programme, as well as potential predictors and consequences on the students' performance and well-being. The strength of this study is that it focuses on students' experiences and interpretations of their educational context. In implying that ambiguity makes it difficult for students to establish whether they have performed "enough" and when it is time to say "no" or harness their ambition, we have established a line of questioning that not only focuses on a required high minimum effort but also on psychological factors that influence how this minimum is experienced and legitimised. 


\section{Acknowledgements}

We thank Professor Lars Dahlgren, Department of Sociology, Umeå University, for contributing his considerable knowledge and expertise in Grounded Theory methodology to this study. 


\section{References}

Bandura, A 1997, Self-efficacy - the exercise of control, W. H. Freeman and Company, New York.

Bussey, K \& Bandura, A 1999, 'Social cognitive theory of gender development and differentiation', Psychological Review, vol. 106, pp. 676-713.

Cassell, J 1997, 'Doing gender, doing surgery: women surgeons in a man's profession', Human Organization, vol. 56, pp. 47-52.

Chambel, JC \& Curral, L 2005, 'Stress in academic life: work characteristics as predictors of student well-being and performance', Applied Psychology: An International Review, vol. 54, pp. 135-47.

Charmaz, K 2003, 'Grounded theory: objectivist and constructivist methods', in NK Denzin \& YS Lincoln (eds.), Strategies of qualitative inquiry, Sage, Thousand Oaks, CA. pp. 249-91.

Charmaz, K 2006, Constructing grounded theory: a practical guide through qualitative analysis, Sage, London, UK.

Connell, RW 2002, Gender, Polity Press, Cambridge, UK.

Cotton, SJ, Dollard, MF \& de Jonge, J 2002, 'Stress and student job design: satisfaction, well-being, and performance in university students', International Journal of Stress Management, vol. 9, pp. 147-62.

Courtenay, WH 2000, 'Constructions of masculinity and their influence on men's well-being: a theory of gender and health', Social Science \& Medicine, vol. 50, pp. 1385-401.

Cutcliffe, JR 2000, 'Methodological issues in grounded theory', Journal of Advanced Nursing, vol. 31, pp. 1476-84.

Davies, K 2001, Disturbing gender: on the doctor-nurse relationship (Lund Studies in Sociology Vol. 4), Lund University, Lund, Sweden.

Divaris, K, Barlow, PJ, Chendea, SA, Cheong, WS, Dounis, A, Dragan, IF et al. 2008, 'The academic environment: the students' perspective', European Journal of Dental Education, vol. 12, suppl. 1, pp. 120-30.

Eriksson, K 2003, Manligt läkarskap, kvinnliga läkare och normala kvinnor: Köns- och läkarskapande symbolik, metaforik och praktik. [Physicianship, female physicians and normal women. Symbolism, metaphors, and practices in the construction(s) of gender and physicians], Doctoral Dissertation, University of Uppsala. Stehag: Förlags ab Gondolin \& Author, Sweden.

Freeman, R, Gorter, R, McWilliams, C \& Williams, S 2007, 'A qualitative exploration of the role of gender, ethnicity and clinical experience 
upon dental students' interpersonal skills when interacting with dental nurses', European Journal of Dental Education, vol. 11, pp. 168-76.

Gamarnikow, E 1978, 'Sexual division of labour: the case of nursing', in A Kuhn \& AM Wolpe (eds.), Feminism and materialism. Women and modes of production, Routledge, London. pp. 96-123.

Glaser, BG 1978, Theoretical sensitivity: advances in the methodology of grounded theory, Sociology Press, Mill Valley, Calif.

Glaser, BG 2002, 'Constructivist grounded theory?' Forum: Qualitative Sozialforschung/Forum: Qualitative Social Research [On-line journal], vol. 3, viewed 12 February 2008, http://www.qualitativeresearch.net/fqs/fqs-eng.htm

Hattie, J \& Timperley, H 2007, 'The power of feedback', Review of Educational Research, vol. 77, pp. 81-112.

Henwood, K \& Pidgeon, N 2003, 'Grounded theory in psychological research', in PM Camic, JE Rhodes \& L Yardley (eds.), Qualitative research in psychology - expanding perspectives in methodology and design, American Psychological Association, Washington, DC. 131-55.

Henzi, D, Davis, E, Jasinevicius, R \& Hendricson, W 2006, 'North American dental students' perspectives about their clinical education', Journal of Dental Education, vol. 70, pp. 361-77.

Henzi, D, Davis, E, Jasinevicius, R \& Hendricson, W 2007, 'In the students' own words: what are the strengths and weaknesses of the dental school curriculum?', Journal of Dental Education, vol. 71, pp. 632-45.

Henzi, D, Davis, E, Jasinevicius, R, Hendricson, W, Cintron, L \& Isaacs, M 2005, 'Appraisal of the dental school learning environment: the students' view', Journal of Dental Education, vol. 69, pp. 1137-47.

Humphris, G, Blinkhorn, A, Freeman, R, Gorter, R, Hoad-Reddick, G, Murtomaa, $\mathrm{H}$ et al. 2002, 'Psychological stress in undergraduate dental students: baseline results from seven European dental schools', European Journal of Dental Education, vol. 6, pp. 22-9.

Jones, S \& Myhill, D 2004, “Troublesome boys” and "compliant girls”: gender identity and perceptions of achievement and underachievement', British Journal of Sociology of Education, vol. 25, pp. 547-61.

Karasek, R \& Theorell, T 1990, Healthy work: stress, productivity, and the reconstruction of working life, Basic Books, New York. 
Nunn, J, Freeman, R, Anderson, E, Carneiro, LC, Carneiro, MSA, Formicola, A, et al. 2008, 'Inequalities in access to education and healthcare’, European Journal of Dental Education, vol. 12, pp. 30-9.

Polychronopoulou, A \& Divaris, K 2005, 'Perceived sources of stress among Greek dental students', Journal of Dental Education, vol. 69, pp. 687-92.

Rosenberg, HM, Cucchiara, AJ \& Helpin, ML 1998, 'Dental students' attitude to gender roles', Social Science \& Medicine, vol. 47, pp. 1877-80.

Statistics Sweden 2007, 'Statistics specially ordered for this research in the spring of 2007', Swedish National Agency for Education [Statistical Database], viewed 4 March 2008, http://www.skolverket.se/sb/d/1721

Swedish National Agency for Higher Education 2007, 'Studentspegeln' [The Student Mirror], Report No 2007:20 R, viewed 12 February 2008, http://www.hsv.se/download/18.5b73fe55111705b51fd80002526/ 0720R.pdf

Umeå dentistry programme - study programme (implemented January 2006), viewed 30 May 2008,

http://www.odont.umu.se/utbildning/tlak/kursplan/utbildningsplan_tla kare.html

West, C \& Zimmerman, DH 1987, 'Doing gender', Gender \& Society, vol. 1, pp. 125-51.

Zillén, PA \& Mindak, M 2000, 'World dental demographics', International Dental Journal, vol. 50, pp. 194-234. 


\section{Tables}

Table 1. The category Experiencing pressure and stress - codes and subcategories

\section{Category: Experiencing pressure and stress}

Subcategories: Managing your time/Catching up

To manage/Standing it

Experiencing inner and outer demands

\section{Codes}

Active responsibility expected All that surrounds it

Being powerless or without a choice Due to organisational flaws

From inexperience or ignorance Prioritising curricular performance Related to stress/time pressure To be judged

To make judgements

Experienced effort needed/burden of work

Has perspective from (working) life experience

Maturing/becoming experienced

Prioritising/structuring your time

Sacrificing your leisure time

To manage/catch up

Having a say in decisions

Prioritising/structuring your time

Programme culture

To maintain a stiff upper lip

To seek/receive support

Experiencing inner demands Experiencing outer demands

Has perspective from (working) life experience

Rising levels

The clinic makes the theoretical studies relevant

The responsibility and power of the teachers 


\section{Figures}

Figure 1. Umeå dental students' experiences of their education, the proposed model

Overall experience

\section{Experiencing ambiguity}

\section{Core category}

\begin{tabular}{|c|c|c|c|c|c|c|c|c|}
\hline $\begin{array}{c}\text { Experiencing } \\
\text { pressure } \\
\text { and stress }\end{array}$ & $\begin{array}{c}\text { Assessing } \\
\text { your own } \\
\text { performance }\end{array}$ & $\begin{array}{c}\text { Passing through } \\
\text { the eye } \\
\text { of the needle }\end{array}$ \\
\hline
\end{tabular}

Categories

Areas of struggle

performance

of the needle

\begin{tabular}{l|c|}
\begin{tabular}{l|c} 
Reflexions \\
on (possible)
\end{tabular} & $\begin{array}{c}\text { Managing your } \\
\text { timel } \\
\text { actions }\end{array}$ \\
Catching up
\end{tabular}

categories 